\title{
Solar Activity Prediction via Artificial Neural Networks applied to X-ray Flux Data
}

\author{
Victor da S. Pedrazzi (IC), Guilherme P. Coelho (PQ), Ana E. A. da Silva (PQ).
}

\begin{abstract}
This research intends to evaluate the use of artificial neural networks to forecast the solar x-ray flux time series, in order to predict the occurrence of solar flares. So far, the network is capable of predicting one minute ahead into the time series with good accuracy.
\end{abstract}

Key words: neural networks, solar activity, prediction

\section{Introduction}

Solar activity comprehends many phenomena which became subject of scientific research due to their potential negative impacts on the Earth's environment. Solar flares, while not being the most dangerous events, often precede the highly harmful but not so frequent Coronal Mass Ejections (CMEs) ${ }^{1}$.

Such intense solar activity can damage technological products, such as power grids, by causing overheating in sensitive equipment ${ }^{2}$. Solar flares of different intensities are associated with distinct solar X-ray flux levels, which are measured in $\left(\mathrm{W} / \mathrm{m}^{2}\right)$ and obtained from the GOES-15 satellite ${ }^{3}$.

Given the context above, this research intends to use the solar X-ray flux data to train and evaluate an artificial network that predicts this same time series, in an attempt to foresee the occurrence of solar flares.

\section{Results and Discussion}

To aid the development of the artificial neural network, the machine learning framework Encog has been used in this research ${ }^{4}$. Many different network configurations have been tested. After a model with a certain degree of accuracy has been identified, we ran the training and evaluation steps 10 times in order to compare the results of different executions of the same prediction model considering the root mean squared error (RMSE) of the predictions, as summarized in Chart 1. For the execution that provided the lowest error, we plotted a line graph comparing the real series with the network's output, as shown in Image 1.

Chart 1. Mean and standard deviation of the evaluation RMSE of all executions

\begin{tabular}{|c|c|c|c|}
\cline { 2 - 4 } \multicolumn{1}{c|}{} & $\mathbf{t + 1}$ & $\mathbf{t + 1 5}$ & $\mathbf{t}+\mathbf{3 0}$ \\
\hline Mean & $2.98 \mathrm{E}-07$ & $1.76 \mathrm{E}-06$ & $1.88 \mathrm{E}-06$ \\
\hline Std. Dev. & $1.93 \mathrm{E}-07$ & $3.37 \mathrm{E}-07$ & $3.49 \mathrm{E}-07$ \\
\hline
\end{tabular}

Image 1. Series of one-minute ahead $(t+1)$ predictions for solar X-ray flux in 12/26/2014

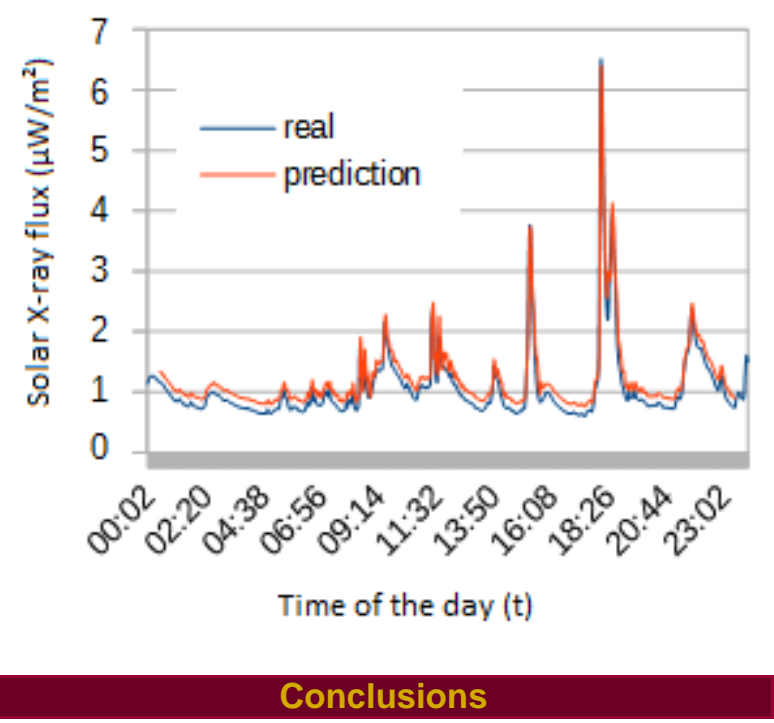

It's noticeable from Image 1 that the network produced outputs similar to the actual data when it comes to prediction of the next step in the time series (one minute ahead forecast). With respect to the predictions in $t+15$ and $t+30$, we observe from Chart 1 that the errors have increased significantly, which indicates that further investigation is still required before the framework can be adopted in such context.

\section{Acknowledgement}

The authors would like to thank CNPq for the financial support.

\footnotetext{
${ }^{1}$ Youssef, M. NRIAG Journal of Astronomy and Geophysics 2012, 1, 172-178.

${ }^{2}$ Metatech. Geomagnetic storms and their impacts on the US power grid; Goleta, CA, 2015.

${ }^{3}$ Rowlett, R. Solar Flare Intensity http://goo.gl/x5HwXw (accessed Jan 25, 2015).

${ }^{4}$ Heaton, J. Encog Machine Learning Framework | Heaton Research http://goo.gl/NTRlQf (accessed Jan 30, 2015).
} 